\title{
The Study of Intervals Sorting Method Based on close degree
}

\author{
Er-dun Bai ${ }^{1}$, Yu-e Bao ${ }^{2, \text { a }}$ \\ ${ }^{1}$ College of Computer Science and Technology, Inner Mongolia University for Nationalities, Tongliao \\ 028043, China \\ ${ }^{2}$ College of Mathematics, Inner Mongolia University for Nationalities, Tongliao 028043, China \\ abyebed@163.com
}

\begin{abstract}
Against the selection of best option, the sorting of programs and other issues, we discuss the sorting issue of interval numbers based on close degree. Then we prose a sorting method of interval numbers based on close degree. Though an example, we verify the rationality and effectiveness of the sorting methods.
\end{abstract}

Keywords: interval number; close degree; sorting method.

\section{Introduction}

In the multi-attribute decision making, due to the complexity of objective things, the finiteness of human knowledge and ability, a lot of decision-making information in the form of interval numbers to represent is more reasonable. Interval multi-attribute decision making usually is converted to interval scheduling problem [1]. However, because the difference of dimension and type of each attribute index values, we cannot directly use the initial index interval values comparing and sorting programs, you need to remove the incommensurability and contradiction between each index value. So, the decision-making index values are normalized to interval number on $[0,1]^{[2-5]}$. Therefore, it has great significance that sort reasonably the interval number on [0,1]. In paper [6], we give the general representation of close degree between interval numbers, and illustrates that we can construct a variety of nearness according to the general representation. On this basis, this paper discusses the sorting indicators of interval number $[0,1]$, and gives a sorting method of interval numbers based close degree.

\section{Preliminary}

Let $R$ denote all the real numbers. For arbitrarily $a^{L}, a^{U} \in R, a^{L} \leq a^{U}$, we call $\left[a^{L}, a^{U}\right]$ is a interval number, i.e. $a=\left[a^{L}, a^{U}\right]$.

If $0 \leq a^{L} \leq a^{U}$, then $a=\left[a^{L}, a^{U}\right]$ is a non-negative interval number. The whole interval numbers on $R$ denote by $[R]$, the whole interval numbers on $[0,1]$ denote by $[I]$, then $[I] \subset[R]$.

Theorem $1.1^{[7]}$ Set $a, b \in[R]$,

$d(a, b)=\sqrt{\left(\frac{a^{L}+a^{U}}{2}-\frac{b^{L}+b^{U}}{2}\right)^{2}+\frac{1}{12}\left[\left(a^{U}-a^{L}\right)-\left(b^{U}-b^{L}\right)\right]^{2}}$,

Then

$$
N(a, b)=\left\{\begin{array}{rc}
\frac{1}{\sqrt{1+d(a, b)},} & d(a, b) \in[0,1) \\
0, & d(a, b) \geq 1
\end{array},\right.
$$

is the close degree of $a$ and $b$. 


\section{The sorting method of interval numbers based on close degree}

Set $a_{i}=\left[a_{i}^{L}, a_{i}^{U}\right](i=1,2, \cdots, n)$ are non-negative interval numbers, through some appropriate normalized such as specific gravity variation method in paper [5], we get the interval numbers on $[0,1]$, $r_{i} \in[I](i=1,2, \cdots, n)$. The steps of sorting them are as:

Step 1: According to the actual situation, we select positive and negative ideal interval numbers using appropriate methods. The usual conventional method is following. The positive ideal interval number of $r_{i}=\left[r_{i}^{L}, r_{i}^{U}\right], i=1,2, \cdots, n$. is

$$
r^{+}=\left[\max \left\{r_{1}^{L}, r_{2}^{L} \cdots r_{n}^{L}\right\}, \max \left\{r_{1}^{U}, r_{2}^{U} \cdots r_{n}^{U}\right\}\right] \text {. }
$$

The negative ideal interval number is

$$
r^{-}=\left[\min \left\{r_{1}^{L}, r_{2}^{L} \cdots r_{n}^{L}\right\}, \min \left\{r_{1}^{U}, r_{2}^{U} \cdots r_{n}^{U}\right\}\right] \text {. }
$$

Step 2: Calculating the close degree $N\left(r_{i}, r^{+}\right), N\left(r_{i}, r^{-}\right)$according to close degree formula. On this basis, according to the idea that the program is better if the distance with positive ideal solution is smaller and with negative ideal solution is greater, we give sorting index formula

$$
T\left(r_{i}\right)=\frac{N\left(r_{i}, r^{+}\right)+\left(1-N\left(r_{i}, r^{-}\right)\right)}{2},
$$

And find the corresponding values of $T\left(r_{i}\right)$.

Step 3: Sorting them according to the size of $T\left(r_{i}\right)$. The value of $T\left(r_{i}\right)$ is greater, the corresponding $a_{i}$ is more front.

\section{Application examples}

Example 3.1 Paper [4] gives the comprehensive evaluation interval values of each program. The data are as follows:

$$
\begin{array}{ll}
r_{1}=[0.1890,0.1976] ; & r_{2}=[0.2022,0.2154] ; \\
r_{3}=[0.2021,0.2112] ; & r_{4}=[0.1865,0.1964] ; \\
r_{5}=[0.1888,0.1983] . &
\end{array}
$$

We sort the comprehensive evaluation interval values.

Step 1 The positive ideal interval number is

$$
\begin{aligned}
& r^{+}=\left[\max \left\{r_{1}^{L}, r_{2}^{L} \cdots r_{5}^{L}\right\}, \max \left\{r_{1}^{U}, r_{2}^{U} \cdots r_{5}^{U}\right\}\right] \\
& =[0.2022,0.2154] \\
& \text { and the negative ideal interval number is } \\
& r^{-}=\left[\min \left\{r_{1}^{L}, r_{2}^{L} \cdots r_{5}^{L}\right\}, \min \left\{r_{1}^{U}, r_{2}^{U} \cdots r_{5}^{U}\right\}\right] \\
& =[0.1865,0.1964] .
\end{aligned}
$$

Step 2 Using formula (1), we get

$$
\begin{aligned}
d\left(r_{1}, r^{+}\right) & =\sqrt{\left[\frac{(0.1890+0.1976)}{2}-\frac{(0.2154+0.2022)}{2}\right]^{2}+\frac{1}{3}\left[\frac{(0.1976-0.1890)}{2}-\frac{(0.2154-0.2022)}{2}\right]^{2}} \\
& =\frac{1}{2} \sqrt{[(0.1890+0.1976)-(0.2154+0.2022)]^{2}+\frac{1}{3}[(0.1976-0.1890)-(0.2154-0.2022)]^{2}} \\
& =\frac{1}{2} \sqrt{(0.3866-0.4176)^{2}+\frac{1}{3}(0.0086-0.0132)^{2}} \\
& =\frac{1}{2} \sqrt{0.031^{2}+\frac{1}{3} \times 0.0046^{2}} \\
& =0.0156
\end{aligned}
$$


In the same way we get

$d\left(r_{1}, r^{-}\right)=0.0014$.

Therefore using formula (2), we get

$$
\begin{aligned}
& N\left(r_{1}, r^{+}\right)=\frac{1}{\sqrt{1+d\left(r_{1}, r^{+}\right)}} \\
& =\frac{1}{\sqrt{1+0.0156}} \\
& =0.9922, \\
& N\left(r_{1}, r^{-}\right)=\frac{1}{\sqrt{1+d\left(r_{1}, r^{-}\right)}} \\
& =\frac{1}{\sqrt{1+0.0014}}, \\
& =0.9993 .
\end{aligned}
$$

Using formula (3), we obtain the sorting index values

$$
\begin{aligned}
& T\left(r_{1}\right)=\frac{N\left(r_{1}, r^{+}\right)+\left(1-N\left(r_{1}, r^{-}\right)\right)}{2} \\
& =\frac{0.9922+(1-0.9993)}{2} \\
& =0.49325 .
\end{aligned}
$$

The same can be other sort index values:

$$
\begin{aligned}
& T\left(r_{2}\right)=0.50855 ; T\left(r_{3}\right)=0.506 ; \\
& T\left(r_{4}\right)=0.49145 ; T\left(r_{5}\right)=0.49345 .
\end{aligned}
$$

Step 3 Sorting them according to the size of $T\left(r_{i}\right)$. The sorting results is

$$
r_{2} \succ r_{3} \succ r_{5} \succ r_{1} \succ r_{4} \text {, }
$$

That is

Program $2 \succ$ program $3 \succ$ program $5 \succ$ program $1 \succ$ program 4 .

So, scheme 2 is the best option.

The result is consistent with literature [4]. However, the method1need a secondary comparison, and method 2 also need to give risk coefficient. The calculation process of method we present is relatively simple, easy and practical.

Example 3.2 We use the Example in [8]. For getting the "Service Star", a bus company investigate the company's five buses $L_{1}, L_{2}, L_{3}, L_{4}, L_{5}$ through questionnaires and other forms, and get the comprehensive evaluation interval values of passenger satisfaction

$$
\begin{aligned}
& r_{1}=[0.8024,0.8110] ; r_{2}=[0.7846,0.7978] ; \\
& r_{3}=[0.7888,0.7971] ; r_{4}=[0.8036,0.8135] ; \\
& r_{5}=[0.8017,0.8112] .
\end{aligned}
$$

The final results in [8] is

$$
r_{1} \succ r_{4} \succ r_{5} \succ r_{3} \succ r_{2} \text {, }
$$

That is

bus $L_{1} \succ$ bus $L_{4} \succ$ bus $L_{5} \succ$ bus $L_{3} \succ$ bus $L_{2}$.

So $L_{1}$ obtain the "Service Star". However, from the point of interval data, the comprehensive evaluation value of $r_{4}$ is better than $r_{1}$.

So, the results are not realistic.

Using our method, we get

$$
T\left(r_{1}\right)=0.5069 ; T\left(r_{2}\right)=0.4917 \text {; }
$$


$T\left(r_{3}\right)=0.4938 ; T\left(r_{4}\right)=0.5087$

$T\left(r_{5}\right)=0.5067$.

Thus, the sorting results is

$r_{4} \succ r_{1} \succ r_{5} \succ r_{3} \succ r_{2}$

That is

bus $L_{4} \succ$ bus $L_{3} \succ$ bus $L_{5} \succ$ bus $L_{1} \succ$ bus $L_{2}$.

So, $L_{4}$ obtain the "Service Star". This result is more in line with the objective reality.

\section{Summary}

Close degree is an important concept of uncertainty mathematical theory, and has been widely applied in solving practical problems. Against the selection of best option, the sorting of programs and other issues, we give the interval number sorting method based on close degree. Firstly, according to comprehensive evaluation interval values of various programs, we determine the positive and negative ideal interval values. Secondly, we calculate the close degree between comprehensive evaluation interval numbers of per program and the positive and negative ideal solution interval numbers. Then, according to the idea that the program is better if the distance with positive ideal solution is smaller and with negative ideal solution is greater, we give sorting index formula of each program. Finally, we calculate the sorting index values of each program, and sort them according to the size of sorting index values. Examples show that the sorting method of interval numbers based on close degree is a reasonable and practical sorting method.

\section{Acknowledgments}

This work is supported by the Inner Mongolia Natural Science Foundation of China (2014MS0107).

\section{References}

[1] Zhang Quan, Fan Zhiping, Pan Dehui. A Ranking Approach with Possibilities for Multiple Attribute Decision Making Problems with Intervals. Control and decision, 1(1999):703-714

[2] Yun-shiow Chen. Fuzzy Ranking and Quadratic Fuzzy Regression [J]. Computers and Mathematics with Applications, 38(1999):265-279.

[3] Yun-shiow Chen. Fuzzy Ranking and Quadratic Fuzzy Regression [J]. Computers and Mathematics with Applications, 38(1999):265-279.

[4] Wang Dian-xuan, Li Zhi-shi. Contrast Coefficient Method of Multi-objective Decision [J].Systems Engineering-Theory\&Practice. 2(1988):66-67

[5] Peng Xiao-qin,Bao Yu-e,Zhao Bo.The Research on the Attribute Normalization for Multiple Attribute Decision Making of Interval Number[J].Journal of Inner Mongolia University for Nationalities,2(2013):129-134.

[6] Peng Xiao-qin, Bao Yu-e,Zhao Bo. Research on the General Expressions of Similarity Measure for Interval Numbers. Mathematics in Practice And Theory 22(2014)258-262.

[7] Bao Yu-e,Guo Li,Liu Guo-feng. A Studuy of Water Environment Quality Evaluation Model Based on Interval Number Closeness [J].Mathematics In Practice and Theory 8 (2014):34-41.

[8] Lan Ji-bin, Hu Ming-ming, Ye Xin-miao. Ranking interval numbers based on similarity [J]. Computer Engineering and Design. 4 (2011):1419-1421. 